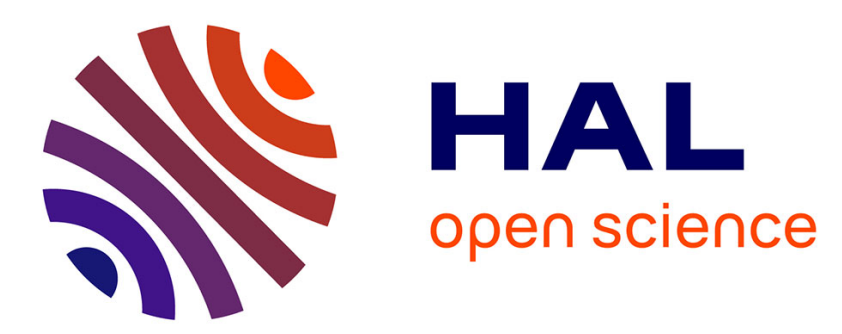

\title{
A computational strategy for geometry optimization of ionic and covalent excited states, applied to butadiene and hexatriene
}

Martial Boggio-Pasqua, Michael J. Bearpark, Michael Klene, Michael A. Robb

\section{- To cite this version:}

Martial Boggio-Pasqua, Michael J. Bearpark, Michael Klene, Michael A. Robb. A computational strategy for geometry optimization of ionic and covalent excited states, applied to butadiene and hexatriene. Journal of Chemical Physics, 2004, 120, pp.7849-7860. 10.1063/1.1690756 . hal-02465986

\section{HAL Id: hal-02465986 \\ https://hal.science/hal-02465986}

Submitted on 4 Feb 2020

HAL is a multi-disciplinary open access archive for the deposit and dissemination of scientific research documents, whether they are published or not. The documents may come from teaching and research institutions in France or abroad, or from public or private research centers.
L'archive ouverte pluridisciplinaire HAL, est destinée au dépôt et à la diffusion de documents scientifiques de niveau recherche, publiés ou non, émanant des établissements d'enseignement et de recherche français ou étrangers, des laboratoires publics ou privés. 


\title{
A computational strategy for geometry optimization of ionic and covalent excited states, applied to butadiene and hexatriene
}

\author{
Martial Boggio-Pasqua, Michael J. Bearpark, Michael Klene, and Michael A. Robb \\ Department of Chemistry, Imperial College London, South Kensington campus, \\ London SW7 2AZ, United Kingdom
}

(Received 19 November 2003; accepted 6 February 2004)

\begin{abstract}
We propose a computational strategy that enables ionic and covalent $\pi \pi^{*}$ excited states to be described in a balanced way. This strategy depends upon (1) the restricted active space self-consistent field method, in which the dynamic correlation between core $\sigma$ and valence $\pi$ electrons can be described by adding single $\sigma$ excitations to all $\pi$ configurations and (2) the use of a new conventional one-electron basis set specifically designed for the description of valence ionic states. Together, these provide excitation energies comparable with more accurate and expensive $a b$ initio methods-e.g., multiconfigurational second-order perturbation theory and multireference configuration interaction. Moreover, our strategy also allows full optimization of excited-state geometries-including conical intersections between ionic and covalent excited states-to be routinely carried out, thanks to the availability of analytical energy gradients. The prototype systems studied are the cis and trans isomers of butadiene and hexatriene, for which the ground $1 \mathrm{~A}_{1 / \mathrm{g}}$, lower-lying dark (i.e., symmetry forbidden covalent) $2 \mathrm{~A}_{1 / \mathrm{g}}$ and spectroscopic $1 \mathrm{~B}_{2 / \mathrm{u}}$ (valence ionic) states were investigated. (C) 2004 American Institute of Physics. [DOI: 10.1063/1.1690756]
\end{abstract}

\section{INTRODUCTION}

The fundamental importance of being able to obtain accurate theoretical descriptions of electronically excited states has been acknowledged since quantum mechanics was applied to chemistry, and a wide range of theoretical methods have been developed for the treatment of electronically excited states of atoms and molecules. For computing these states, multireference methods are usually necessary for a qualitatively correct representation of the wave function. Furthermore, obtaining accurate electronic excitation energies usually requires an additional high-level treatment of dynamic electron correlation effects. Unfortunately, this treatment is likely to be expensive computationally: the most accurate methods tend to be the most time consuming and only presently applicable to systems that contain a few light atoms. Another major problem with these highly correlated methods at present is that most lack analytical energy gradients for geometry optimizations. This is a severe limitation, as the gradient has to be computed numerically, which becomes more expensive and less accurate as the number of atoms increases. This makes geometry optimizations only possible for very small systems, and extensive study of potential energy surfaces (PESs) expensive, if not unworkable.

On the other hand, several computational methods for excited states developed in recent years combine reasonable accuracy with speed sufficient to allow one to treat systems containing up to a dozen heavy atoms. Some of these methods have analytical energy gradients, which make them practical to use for investigating the topology of excited-state PESs. Unfortunately, these methods usually offer a poor description of the dynamic electron correlation so important for ionic excited states. This is the case with the complete active space self-consistent field (CASSCF) method in particular, which includes only the static (long-range, nondynamic) correlation by default. The CASSCF method focuses on just a few "active" electrons, in active orbitals chosen to give multiple configurations describing the required electronic state. Unfortunately, correlating only the active electrons often gives energies that are quantitatively incorrect. For reliable calculations of ionic excited states, one must go beyond the CASSCF level and include dynamic correlation between active and inactive electrons.

In this article, we propose a computational strategy based on CASSCF that enables ionic and covalent $\pi \pi^{*}$ excited states to be described in a balanced way, providing excitation energies comparable with more accurate and expensive $a b$ initio methods-e.g., multiconfigurational second-order perturbation theory (CASPT2) and the multireference configuration interaction (MRCI). This strategy depends upon (1) the restricted active space self-consistent field (RASSCF) method, in which the dynamic correlation between the core $\sigma$ and valence $\pi$ electrons is included by adding single $\sigma$ excitations to all $\pi$ configurations, and (2) the use of a new conventional one-electron basis set specifically designed for the description of valence ionic states. Full optimization of excited state geometries and conical intersections (CIs) between ionic and covalent excited states can now be routinely carried out with RASSCF gradients.

The prototype systems chosen to test this new strategy are butadiene and hexatriene, for which dynamic electron correlation effects are well known to be crucial. These polyenes have been the subject of numerous spectroscopic investigations, because of their importance in the photoconversion of biomolecules, photochromic materials, and photoconductivity. Despite their structural simplicity, they still represent a challenge for electronic structure theory. 
Two main problems are encountered when computing the electronic structures of polyenes. First, both valence and Rydberg excited states are present, and the mixing between these states can be significant in the lowest-lying $\pi \rightarrow \pi^{*}$ ionic excited states. Second, the energetic ordering of the two lowest excited states $2{ }^{1} \mathrm{~A}_{1 / \mathrm{g}}(2 \mathrm{~A})$ and $1{ }^{1} \mathrm{~B}_{2 / \mathrm{u}}(1 \mathrm{~B})$ is a well-known difficulty, because these states have a rather different character and cause different problems for different computational methods. Previous studies on the title systems agree that the $2 \mathrm{~A}$ states are of valence-covalent nature and exhibit a large double-excitation character: $|\mathrm{HOMO}, \mathrm{HOMO}\rangle \rightarrow \mid$ LUMO,LUMO $\rangle$. These are "dark" or "phantom" states as they have low absorption cross sections. On the other hand, the spectroscopically allowed 1B states are singly excited valence ionic states dominated by $|\mathrm{HOMO}\rangle \rightarrow|\mathrm{LUMO}\rangle$.

Numerous calculations have been performed on the two lowest excited $1 \mathrm{~B}$ and 2A states of butadiene and hexatriene. Accurate $a b$ initio methods including CASPT2 (Refs. 1 and 2) multireference Møller-Plesset (MRMP) (Ref. 3), and MRCI (Refs. 4-7) have been used to calculate the vertical excitation energies of these states. Linear-response coupledcluster (LR-CC) methods have also been employed ${ }^{8}$ and, more recently, quadratic response theory has been applied in conjunction with the self-consistent field (QR-SCF) method and a hierarchical set of coupled-cluster methods (QR-CC). ${ }^{9}$ Time-dependent density functional theory (TDDFT) has also been used to study the vertical excitation energies of alltrans linear polyenes. ${ }^{10}$

To our knowledge, only Page and Olivucci ${ }^{11}$ have performed CASPT2 geometry optimizations on butadiene, using numerical energy gradients. Similar computations are currently too expensive in the case of hexatriene. Therefore, with this in mind, we have made use of our new RASSCFbased strategy to characterize critical points on the PESs of butadiene and hexatriene, including ionic/covalent conical intersections for butadiene. The choice of orbital spaces, excitations and basis set has been investigated in this work, and the results obtained benchmarked against the most accurate calculations available.

This paper is structured as follows: Section II deals with the computational details: the problem of choosing an adequate set of active orbitals in CASSCF calculations and the construction of a basis set adapted for the description of valence ionic states. In Sec. III, excitation energies are presented for the two isomers of butadiene and for $c Z c$ - and $t E t$-hexatriene, and compared with other high-level ab initio calculations. Ionic/covalent crossing structures are described for butadiene as well. Finally, our conclusions are given in Sec. IV.

\section{COMPUTATIONAL METHODS}

Surprisingly few calculations have been performed using the RASSCF method to date. This is probably due to the difficulty of defining more than one active space of orbitals, as discussed below. To start with, however, we review a method with just one active space: CASSCF.

In the CASSCF approach, orbitals are partitioned into active and spectator sets: active orbitals are those directly involved in the chemical process of interest; spectator orbitals are those that are not. Often, the required active orbitals are a subset of the valence orbitals. A CASSCF wave function is built by distributing active electrons in all consistent ways among the active orbitals, converting the problem of choosing many-electron configuration-state functions (CSFs) into the problem of choosing active orbitals. All orbitalsspectator and active - are fully optimized in the calculation, but correlation energy is only recovered for the active electrons.

The choice of active space is therefore crucial if the CASSCF method is to correctly describe electron redistribution during the chemical process of interest. This choice can be difficult, particularly for any excited states involving orbitals outside of the valence space (e.g., Rydberg orbitals). Aside from the trial and error procedure necessary to select the correct active orbitals, the number of electron configurations increases factorially as orbitals are added to the active space, which may make the desired calculation prohibitively expensive.

To date, the most successful computations of vertical excitation energies using this type of method have been carried out with Roos' restricted active space (RAS) configuration selection. ${ }^{12,13}$ Typically, only one or two electrons populate the orbitals added from outside the valence space in any important electron configuration. Many-electron configurations (which would be included in a full CASSCF calculation) are therefore largely redundant. Recognizing this, the spirit of the RASSCF method is to subdivide the active space into three categories: orbitals with a limited number of vacancies (called the RAS1 space), a fully active orbital set (RAS2), and orbitals with a limited number of electrons (RAS3). By eliminating the redundant configurations, the size of the configuration interaction problem can be greatly reduced with RASSCF compared to CASSCF without compromising accuracy. Calculations that include a large number of orbitals outside the valence space therefore become feasible. Nonetheless, the choice of the RAS1 and RAS3 active space, in general, is not often intuitive.

Further details about RASSCF can be found elsewhere. ${ }^{12}$ The abbreviation $(n, m)$ is used in this article to define the number of active electrons $(n)$ and active orbitals $(m)$. The notation $\left(n, m^{\mathrm{I}}+m^{\mathrm{II}}+m^{\mathrm{III}}\right)[h, e]$ is used for specifying the type of RASSCF; $m^{\mathrm{I}}, m^{\mathrm{II}}$, and $m^{\mathrm{III}}$ defining the number of active orbitals in RAS1, RAS2, and RAS3 spaces, respectively. The values of $h$ and $e$ specify the number of holes in RAS1 and electrons in RAS3, respectively, defining in this way the restriction of the type of excitations between the different active spaces.

In this paper, we start with CASSCF calculations including all valence $\pi$ orbitals in the active space for butadiene and hexatriene. For excitation energies, we need to describe the differences in correlation energy between states, and in the standard CASSCF method described above, only the $\pi$ component of the nondynamic correlation is recovered. In order to stabilize the ionic configurations, we first need to recover the $\pi$ component of the dynamic valence-virtual correlation as well, which can be done by extending the $\pi$ active space. This first part of our strategy is not new and has al- 
ready been used in a number of studies. ${ }^{1-6}$ For polyenes, the additional orbitals are more diffuse $3 \pi$-like orbitals (from $3 p$ atomic orbitals of the carbon atoms) with two more nodes (Fig. 1). The active space is then doubled in each symmetry.

The CASSCF calculations described above still use the same core $\sigma$ orbitals for all configurations, preventing the polarization of the core needed to stabilize ionic configurations further. To remedy this flaw, the second part of our strategy is to perform $\sigma \pi$ RASSCF calculations (on transbutadiene and hexatriene) including all of the valence orbitals in the active spaces as well as the $3 \pi$ orbitals. The choice of valence orbitals is justified by the fact that we are only interested in valence states of these systems. For butadiene (22 valence electrons), RAS1 is made up of all the nine occupied $\sigma$ orbitals, RAS2 contains five $\pi$ orbitals (four valence $2 \pi$ plus the most important $3 \pi$ ) and nine $\sigma^{*}$ and the remaining three $3 \pi$ orbitals define the RAS3 space. For hexatriene (32 valence electrons), RAS1 is composed of all 13 occupied $\sigma$ orbitals, RAS 2 contains six valence $2 \pi$ orbitals, and RAS3 is made up of $13 \sigma^{*}$ and six $3 \pi$ orbitals. As shown in a previous study, ${ }^{14}$ the dynamic correlation between $\sigma$ core and $\pi$ electrons can be included by adding single $\sigma$ excitations to all of the $\pi$ configurations. This enables the $\pi$ system to polarize the $\sigma$ framework in the ionic state, thus stabilizing this state relative to the covalent states. Hence only one vacancy and one electron have been allowed in RAS1 and RAS3, respectively. The effects of double excitations have been assessed in the case of butadiene.

The choice of a one-electron basis set for polyenes is complicated because of the possibility of significant valence-Rydberg mixing. Because of our interest in the first three valence states only, we do not require very diffuse basis functions, yet a reasonably extended basis set has to be used in order to describe the dynamic electron correlation arising from the ionic state properly. A range of basis sets was tested, including Pople and co-workers ${ }^{15}$ 6-31G ${ }^{*}$ and $6-31+\mathrm{G}^{*}$ and Dunning's ${ }^{16}$ cc-pVDZ, aug-cc-pVDZ, ccpVTZ, and aug-cc-pVTZ. A new basis set-denoted $6-31 \mathrm{G}^{*}+3 p$ - was generated by adding an explicit $3 p$ function to the standard $6-31 \mathrm{G}^{*}$ basis on each carbon atom. This " $3 p$ function" is the $3 p$ part of the $6-31 \mathrm{G}$ basis set of silicon, which for this split valence basis set consists of a contracted $p$ function with a radial node and an uncontracted $p$ function without. No $s$ functions were added. The $3 p$ exponents were scaled (by factor 0.5 ) so as to stabilize the ionic state in butadiene. This improves the description of the $1 \mathrm{~B}$ ionic states selectively by allowing a better representation of the dynamic correlation of the two electrons occupying the same $p$ orbital.

It is important to note that there are some limitations to the use of such a basis set. As it is designed for describing valence ionic states, it is not appropriate for the treatment of Rydberg states (no true diffuse functions). Thus, in the case of an intruder Rydberg state, our basis set would not be adequate as a mixing between the Rydberg state and the valence state of interest would occur. As a result of this contamination, the valence state would artificially be too low in energy. Nonetheless, this effect is expected to be small, as the Rydberg states cannot be described without true diffuse functions.

In summary, our RASSCF strategy for polyenes consists of three steps:

(1) Double the $\pi$ active space.

(2) Add an explicit $3 p$ function to the carbon atoms.

(3) Include $\sigma \pi$ correlation with RASSCF, further extending the active space.

The ground-state geometries used to calculate the vertical excitation energies of butadiene and hexatriene were optimized at the $\operatorname{CASSCF}(n, n) / 6-31 \mathrm{G}^{*}$ level using the valence $\pi$ orbitals as the active orbitals. Analytical frequency calculations could not always be performed with a full $(n, 2 n)$ double active space, because of the low occupations of some orbitals. Instead, CAS $(4,5)$ frequency calculations had to be performed for some butadiene structures. However, removing the weakly occupied orbitals is not expected to change the nature of the stationary point on the PESs. All of the CASSCF and RASSCF calculations were performed with development versions of GAUSSIAN $99 .{ }^{17}$

\section{RESULTS}

\section{A. Cis-butadiene}

Cis-butadiene has not been studied as intensively as its trans conformer, and information on its excited states is scarce. The maximum assigned to the $1 \mathrm{~A}_{1} \rightarrow 1 \mathrm{~B}_{2}$ transition has been observed at $5.49 \mathrm{eV}^{18}$ As shown in previous works, ${ }^{1,6}$ cis-butadiene is less difficult to treat than transbutadiene from a theoretical point of view because of a lower valence-Rydberg mixing. Consequently, we chose this system to calibrate our CASSCF calculations on the vertical excitation energies of the first two excited states. The most recent theoretical studies include MRCI, ${ }^{4,6}$ CASPT2, valence-shell effective Hamiltonian ${ }^{19}$ (VSEH), and coupledcluster calculations. ${ }^{8}$ The topology and energetics of the potential energy surfaces associated with the $2 \mathrm{~A}_{1}$ and $1 \mathrm{~B}_{2}$ valence excited states have also been investigated at the CASPT2 level of theory using an extended basis set. ${ }^{20}$

\section{Vertical excitation energies}

In this subsection, we discuss the effect of including $3 \pi$ orbitals in the active space and explicit $3 p$ functions in the basis set on the calculated vertical excitation energies of cisbutadiene.

Table I shows that CASSCF using the $(4,4)$ active space gives a poor result as far as the ionic state vertical excitation energy is concerned: about $2.5 \mathrm{eV}$ above the experimental value. Doubling the active space by adding $3 \pi$ orbitals decreases the ionic-state vertical excitation energy by $0.34 \mathrm{eV}$. This stabilization of the ionic state can be explained by the fact that a large part of the $\pi$ component of the dynamic valence-virtual correlation is recovered. Since the vertical excitation energy of the $2 \mathrm{~A}_{1}$ state remains the same, the energy difference between the two excited states is reduced notably as well $(23 \%)$.

Explicitly adding $3 p$ functions to the basis set leads to a dramatic effect. The energy of the ionic state drops considerably $(1.17 \mathrm{eV})$, giving a $1 \mathrm{~A}_{1} \rightarrow 1 \mathrm{~B}_{2}$ vertical transition en- 
TABLE I. Vertical excitation energies of the $1 \mathrm{~B}_{2}$ and $2 \mathrm{~A}_{1}$ states of cisbutadiene. Geometries in Fig. S1 (Ref. 21).

\begin{tabular}{|c|c|c|c|c|c|}
\hline Method & $\begin{array}{c}1 \mathrm{~B}_{2} \Delta E \\
(\mathrm{eV})\end{array}$ & $\left\langle x^{2}\right\rangle$ & $\begin{array}{c}2 \mathrm{~A}_{1} \Delta E \\
(\mathrm{eV})\end{array}$ & $\left\langle x^{2}\right\rangle$ & $\begin{array}{c}\Delta E\left(\mathrm{~B}_{2}-\mathrm{A}_{1}\right) \\
(\mathrm{eV})\end{array}$ \\
\hline $\operatorname{CASSCF}(4,4) / 6-31 \mathrm{G}^{*}$ & 7.97 & 21.8 & 6.63 & 21.0 & 1.34 \\
\hline $\operatorname{CASSCF}(4,8) / 6-31 \mathrm{G}^{*}$ & 7.63 & 21.8 & 6.60 & 21.0 & 1.03 \\
\hline $\operatorname{CASSCF}(4,8) / 6-31+\mathrm{G}^{*}$ & 6.46 & 29.7 & 6.47 & 22.5 & -0.01 \\
\hline $\operatorname{CASSCF}(4,8) / 6-31 \mathrm{G}^{*}+3 p$ & 6.31 & 41.9 & 6.47 & 22.4 & -0.16 \\
\hline $\operatorname{CASSCF}(4,8) / \mathrm{ANO}^{\mathrm{a}}$ & 6.73 & 37.4 & 6.53 & 22.1 & 0.20 \\
\hline $\mathrm{MRCI} / \mathrm{MIDI} 3+2 p^{\mathrm{b}}$ & 6.41 & 33.3 & 6.64 & $\ldots$ & -0.23 \\
\hline $\mathrm{MRCI} / \mathrm{ANO}^{\mathrm{c}}$ & 5.55 & 30.2 & 5.74 & 22.4 & -0.19 \\
\hline CASPT2/ANO & 5.58 & $\ldots$ & 6.04 & $\ldots$ & -0.46 \\
\hline $\mathrm{VSEH}^{\mathrm{d}} \sim$ & 5.62 & $\cdots$ & 6.06 & $\cdots$ & -0.44 \\
\hline $\mathrm{LR}-\mathrm{CCSD}(\widetilde{\mathrm{T}}) / \mathrm{ANO}^{\mathrm{e}}$ & 5.36 & 26.3 & 6.64 & 23.9 & -1.28 \\
\hline Expt. $^{\mathrm{f}}$ & 5.49 & $\cdots$ & $\cdots$ & $\cdots$ & $\ldots$ \\
\hline $\begin{array}{l}{ }^{\mathrm{a}} \text { Reference } 1 . \\
{ }^{\mathrm{b}} \text { Reference } 4 . \\
{ }^{\mathrm{c}} \text { Reference } 6 .\end{array}$ & & $\begin{array}{l}{ }^{\mathrm{d}} \operatorname{Ref} \\
{ }^{\mathrm{e}} \operatorname{Ref} \\
{ }^{\mathrm{f}} \operatorname{Ref}\end{array}$ & $\begin{array}{l}\text { ence } 19 . \\
\text { ence } 8 . \\
\text { ence } 18 .\end{array}$ & & \\
\hline
\end{tabular}

ergy of $6.31 \mathrm{eV}$, only $0.8 \mathrm{eV}$ above the experimental result. Only at this level is the order of states in agreement with other highly correlated approaches-i.e., the $1 \mathrm{~B}_{2}$ ionic state is below the covalent $2 \mathrm{~A}_{1}$ excited state (Table I). It is important to note that the addition of explicit $3 p$ functions in the basis set is more effective than simply adding standard diffuse $s$ and $p$ functions. So although containing fewer functions, our basis set provides a better energy. Note also that $\operatorname{CASSCF}(4,4) / 6-31 \mathrm{G}^{*}+3 p$ would not be adequate as at least five $\pi$ orbitals in the active space are needed for the two excited electronic states to be described simultaneously. ${ }^{4}$

Serrano-Andres et al. ${ }^{1}$ also performed extended CASSCF calculations using a $(4,8)$ active space with a large atomic natural orbital (ANO) basis set. They found the ionic state to be $0.2 \mathrm{eV}$ above the covalent state (Table I). This result can be explained by the fact that the molecular orbitals (MOs) have been obtained from state-averaged CASSCF calculations, where the averaging is over several states of a given symmetry (three states for both ${ }^{1} \mathrm{~A}_{1}$ and ${ }^{1} \mathrm{~B}_{2}$ symmetries). Moreover, the active space is slightly different from ours: five $b_{1}$ and three $a_{2}$ active $\pi$ orbitals, whereas we used four $b_{1}$ and four $a_{2}$ active $\pi$ orbitals. CASPT2 (Ref. 1) and VSEH (Ref. 19) place $1 \mathrm{~B}_{2}$ about $0.45 \mathrm{eV}$ below $2 \mathrm{~A}_{1}$, whereas our best CASSCF result-CAS(4,8)/6$31 \mathrm{G}^{*}+3 p$-gives an energy difference of only $0.16 \mathrm{eV}$. This is in better agreement with the MRCI results of Szalay et al. ${ }^{4}$, which give an energy splitting of about $0.2 \mathrm{eV}$.

We show in this paragraph that it is possible to obtain an "effectively complete" basis set to treat valence ionic states without including too many basis functions. Ab initio calculations using Dunning's correlation consistent basis sets ccpVDZ, aug-cc-pVDZ, cc-pVTZ, and aug-cc-pVTZ have been performed on cis-butadiene (Table S1, available through the EPAPS depository). ${ }^{21}$ Explicit $3 p$ functions on each carbon atom have also been added. When an extra $3 p$ function is added on the carbon atoms $(+3 p)$, there is no need to have another set of diffuse functions (aug) since they do not improve the description of the ionic state. We obtain an identical vertical excitation energy of $6.31 \mathrm{eV}$ for the ionic state using $6-31 \mathrm{G}^{*}+3 p$ (Table $\mathrm{I}$ ) and aug-cc-pVDZ

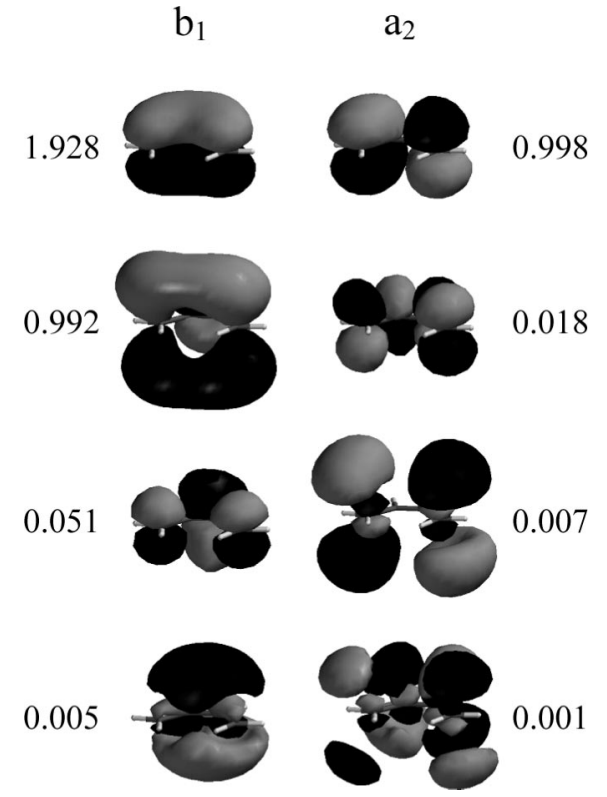

FIG. 1. Active orbitals of the ionic states of cis-butadiene at the $\operatorname{CASSCF}(4,8) / 6-31 \mathrm{G}^{*}+3 p$ level and at the ground-state geometry. The occupation numbers are specified for each orbital.

(Table $\mathrm{S} 1$ ). This result shows that the $6-31 \mathrm{G}^{*}+3 p$ basis set is as accurate as the extended aug-cc-pVDZ basis set for the 1B vertical excitation energy. Hence we have built an "effectively complete" basis set in the sense that it includes the most important basis functions for treating valence ionic states, but not the more diffuse functions included in extended basis sets such as aug-cc-pVDZ, which are less important for ionic states. Indeed, $6-31 \mathrm{G}^{*}+3 p$ contains only 146 primitive Gaussians compared to the 246 in the extended aug-cc-pVDZ. This reduces considerably the computational effort, as the integral code of Gaussian is optimized for Pople-type basis sets and is less efficient in the case of generally contracted basis sets.

Figure 1 shows the eight active orbitals for the $1 \mathrm{~B}_{2}$ state at the ground-state geometry. The shape of the occupied $\pi^{*}$ orbital (second $b_{1}$ orbital) reveals an inner part of valence character combined with a more diffuse Rydberg-type envelope. The spatial extent of this orbital is considerably larger than that of the ground state, but nevertheless is significantly less diffuse than a pure Rydberg state. In other words, the present calculations suggest that this state is of a $\pi \rightarrow \pi^{*}$ type, but the $\pi^{*}$ orbital is somewhat more diffuse than a pure valence orbital. It means that this state has a mixed valence and Rydberg character at the CASSCF level. The expectation value $\left\langle x^{2}\right\rangle$ (with $x$ the axis perpendicular to the plane of the molecule) quantifies the spatial extent of the wavefunction and gives a measure of the diffuse character of the state (Table I). The value of $\left\langle x^{2}\right\rangle$ for the $1 \mathrm{~B}_{2}$ state depends strongly on the ab initio level used (especially on the choice of basis set), much like in the $\mathrm{V}$ state of ethylene. The CASSCF $(4,8) / 6-31 \mathrm{G}^{*}+3 p$ result is computed to be 41.9 a.u. ${ }^{2}$. This is almost double the value obtained at the CASSCF level without the explicit $3 p$ functions in the basis set and agrees well with the CASSCF result of SerranoAndres et al. ${ }^{1}$ The valence-Rydberg mixing is an artifact of 
the CASSCF calculation due to the different correlation effects between valence and Rydberg states. The poor description of the dynamic correlation at this level gives rise to an erroneous and too strong interaction between the valence state and a Rydberg state. Nevertheless, this feature is also observed at other levels of theory like MRCI and CASPT2 that are not able to correct the electron density.

In summary, a double active space, together with explicit $3 p$ functions in the basis set $\left(6-31 \mathrm{G}^{*}+3 p\right)$ appears to be a good choice to describe the order of the first two excited states of cis-butadiene. We therefore chose to use this basis set for all of the following calculations. We expect to improve these results by using the more flexible RASSCF approach to recover $\sigma \pi$ correlation. This has been done for trans-butadiene, for which more theoretical and experimental data are available. Supplementary tables and figures illustrating the topology of the PESs of cis-butadiene are available through the EPAPS depository. ${ }^{21}$

\section{B. Trans-butadiene}

The electronic spectrum of trans-butadiene has been intensively studied through the use of different experimental techniques. ${ }^{22-30}$ A variety of Rydberg states have been identified, and the position of the valence $1 \mathrm{~B}_{\mathrm{u}}$ state, which displays a strong absorption from the ground state, is known to be $5.92 \mathrm{eV}$ (Ref. 29) (vertical) and $5.73 \mathrm{eV}$ (Ref. 31) (adiabatic, $0-0$ ). More recently, though, the $1 \mathrm{~B}_{\mathrm{u}}$ state has been placed at $6.25 \mathrm{eV}$ by McDiarmid. ${ }^{32}$ In the late 1970s, the position of the $2 \mathrm{~A}_{\mathrm{g}}$ covalent state was assigned to a broad, structureless band in the region of $7.3 \mathrm{eV}$ in the electron energy loss spectrum of butadiene. ${ }^{27,33}$ More recently, Raman spectroscopy placed this state at significantly lower energies, in the range $5.4-5.8 \mathrm{eV}^{34,35}$ Nonetheless, there is no evidence concerning the adiabatic or vertical nature of the observed transition. It is worth noting that both the onephoton symmetry forbiddenness and strong doubly excited character complicate locating the $2 \mathrm{~A}_{\mathrm{g}}$ state by absorption spectroscopy. In longer polyenes, this complication is overcome by measuring fluorescence, but no fluorescence is observed for butadiene, making the detection of the state significantly more difficult. Recently, Fuß et al. investigated the ultrafast electronic relaxation from $1 \mathrm{~B}_{\mathrm{u}}$ observed after selective UV excitation of trans-butadiene. ${ }^{36}$

Trans-butadiene has been the subject of numerous theoretical investigations as well. ${ }^{2,3,5,8-10,37-53}$ The structure, vibrational spectra, and force constants have been studied with ab initio Hartree-Fock (HF), Møller-Plesset second-order perturbation (MP2), and Møller-Plesset fourth order perturbation (MP4) calculations using various basis sets. ${ }^{49}$ Excited electronic states have been investigated through a number of different configuration interaction calculations. ${ }^{5,38,39,42,43}$ The CASPT2 method has been employed to calculate the vertical electronic spectrum of trans-butadiene. , $39,51^{2}$ More recently, Cabrero and co-workers used the difference dedicated configuration interaction scheme in the generalized Davidson's correction for the MRCI description to calculate this spectrum. ${ }^{53}$ VSEH calculations have also been performed by Graham and Freed, ${ }^{46}$ while Cave ${ }^{47}$ applied quasidegenerate variational perturbation theory. Watts et al. ${ }^{8}$ used LR-CC- based approaches, while Hsu et al. ${ }^{10}$ applied TDDFT to compute the vertical excitation energies of trans-butadiene. Nakayama and co-workers ${ }^{3}$ used MRMP theory to study the valence $\pi \rightarrow \pi^{*}$ excited states. Lappe and Cave ${ }^{39}$ studied the excitation energy to the $2 \mathrm{~A}_{\mathrm{g}}$ state using a variety of ab initio electronic structure techniques. Cronstrand et al. ${ }^{9}$ applied QR-SCF and QR-CC theories to calculate vertical excitation energies and transition dipole moments between excited electronic states. Finally, Ostojic and Domcke ${ }^{51}$ used CASPT2 to investigate the potential energy surfaces of the $1 \mathrm{~A}_{\mathrm{g}}, 2 \mathrm{~A}_{\mathrm{g}}$, and $1 \mathrm{~B}_{\mathrm{u}}$ electronic states. They identified coordinates along which a crossing between $1 \mathrm{~B}_{\mathrm{u}}$ and $2 \mathrm{~A}_{\mathrm{g}}$ is observed. Based on their CASPT2 surfaces, they constructed a vibronic-coupling model describing the $1 \mathrm{~B}_{\mathrm{u}} / 2 \mathrm{~A}_{\mathrm{g}}$ conical intersection. The actual $1 \mathrm{~B}_{\mathrm{u}}$ lifetime was quantitatively calculated from those surfaces and wave packet dynamics to be $30-40 \mathrm{fs}$, the relaxation leading to the $2 \mathrm{~A}_{\mathrm{g}}$ surface via the conical intersection. This result is in very good agreement with the subsequent experimental observations of $\mathrm{Fu} 3$ et al. $^{36}$

\section{Vertical excitation energies}

As for cis-butadiene, CASSCF calculations using $(4,4)$ and $(4,8)$ active spaces have been performed at the groundstate geometry of trans-butadiene. These results are gathered in Table II along with selected theoretical results. As observed in cis-butadiene, the use of a $(4,8)$ active space combined with the addition of an explicit $3 p$ function in the basis set is needed to describe suitably the relative stability of the ionic and covalent excited states: the $2 \mathrm{~A}_{\mathrm{g}}$ and $1 \mathrm{~B}_{\mathrm{u}}$ states are found to be nearly degenerate, with the ionic state $0.03 \mathrm{eV}$ below the covalent state. This is very different from the CASSCF result of Serrano-Andres et al., ${ }^{2}$ which places the $1 \mathrm{~B}_{\mathrm{u}}$ state surprisingly $8.54 \mathrm{eV}$ above the ground state. The same active space was used in this calculation but the basis set chosen was an ANO basis supplemented with two diffuse $p$ functions on the carbon atoms in order to describe some Rydberg states as well. They found two $\mathrm{B}_{\mathrm{u}}$ Rydberg states below the valence ionic $1 \mathrm{~B}_{\mathrm{u}}$, which suggests that this basis set favors the diffuse Rydberg states to the detriment of the valence $1 \mathrm{~B}_{\mathrm{u}}$ state. We have confirmed this hypothesis by performing similar calculations without the two extra diffuse functions in the ANO basis set. The ionic state (characterized by $\left\langle z^{2}\right\rangle=30.7$ a.u., where $z$ is the axis perpendicular to the plane of the molecule ${ }^{54}$ ) is then placed $7.01 \mathrm{eV}$ above the ground-state energy, in much better agreement with our CASSCF result.

Besides, our CASSCF excitation energies are in very good agreement with a number of earlier MRCI, CASPT2, and MRMP results, which place $1 \mathrm{~B}_{\mathrm{u}}$ below $2 \mathrm{~A}_{\mathrm{g}}$ by an energy ranging from 0.03 to $0.2 \mathrm{eV}$. However, high-level QR$\mathrm{CC} 3$ calculations place the $2 \mathrm{~A}_{\mathrm{g}}$ state $0.5 \mathrm{eV}$ above the ionic state. Furthermore, most of the recent theoretical values are converging on $6.2 \mathrm{eV}$ for the vertical excitation energy of the ionic state, in good agreement with the latest experimental result $(6.25 \mathrm{eV})$. Hence our CASSCF values seem about 0.3 $\mathrm{eV}$ too high for the ionic state. It is still debated whether the most intense peak observed at $5.92 \mathrm{eV}$ corresponds to a vertical or a nonvertical transition. Analysis of the $\mathrm{C}_{2} \mathrm{H}_{4}$ spec- 
TABLE II. Vertical excitation energies of the $1 \mathrm{~B}_{\mathrm{u}}$ and $2 \mathrm{~A}_{\mathrm{g}}$ states of trans-butadiene. Geometries in Table III and Fig. 2.

\begin{tabular}{|c|c|c|c|c|c|}
\hline Method & $1 \mathrm{~B}_{\mathrm{u}} \Delta E(\mathrm{eV})$ & $\left\langle z^{2}\right\rangle$ & $2 \mathrm{~A}_{\mathrm{g}} \Delta E(\mathrm{eV})$ & $\left\langle z^{2}\right\rangle$ & $\begin{array}{c}\Delta E\left(\mathrm{~B}_{\mathrm{u}}-\mathrm{A}_{\mathrm{g}}\right) \\
(\mathrm{eV})\end{array}$ \\
\hline $\operatorname{CASSCF}(4,4) / 6-31 \mathrm{G}^{*}$ & 8.64 & 21.7 & 6.75 & 20.9 & 1.89 \\
\hline $\operatorname{CASSCF}(4,8) / 6-31 \mathrm{G}^{*}$ & 8.32 & 21.6 & 6.76 & 20.9 & 1.56 \\
\hline $\operatorname{CASSCF}(4,8) / 6-31 \mathrm{G}^{*}+3 p$ & 6.56 & 55.7 & 6.59 & 23.8 & -0.03 \\
\hline $\operatorname{RASSCF}(22,9+5+12)[1,1] / 6-31 \mathrm{G}^{*}+3 p$ & 6.50 & 48.1 & 6.99 & 25.0 & -0.49 \\
\hline $\operatorname{RASSCF}(22,9+5+12)[2,2] / 6-31 \mathrm{G}^{*}+3 p$ & 6.60 & 52.0 & 6.93 & 26.0 & -0.33 \\
\hline $\operatorname{CASSCF}(4,8) / \mathrm{ANO}^{\mathrm{a}}$ & 8.54 & 40.9 & 6.64 & 23.2 & 1.90 \\
\hline $\mathrm{MRCI} / \mathrm{MIDI} 3+2 p d^{\mathrm{b}}$ & 6.70 & 54.2 & 6.78 & $\ldots$ & -0.08 \\
\hline $\mathrm{MRCI} / \mathrm{ANO}^{\mathrm{c}}$ & 6.21 & 41.3 & 6.24 & 23.3 & -0.03 \\
\hline CASPT2/ANO & 6.23 & $\ldots$ & 6.27 & $\ldots$ & -0.04 \\
\hline CASPT2/ANO ${ }^{\mathrm{d}}$ & 6.06 & $\ldots$ & 6.27 & $\ldots$ & -0.21 \\
\hline MRMP/QZ3 $p^{\mathrm{e}}$ & 6.21 & $\cdots$ & 6.31 & $\cdots$ & -0.10 \\
\hline $\mathrm{VSEH}^{\mathrm{f}} \sim$ & 6.14 & $\cdots$ & 6.19 & $\cdots$ & -0.05 \\
\hline LR-CCSD $(\widetilde{T}) / \mathrm{ANO}^{\mathrm{g}}$ & 6.13 & 31.7 & 6.76 & 36.1 & -0.63 \\
\hline QR-CC3/cc-pVTZ' $+5^{\mathrm{h}}$ & 6.26 & $\ldots$ & 6.76 & $\ldots$ & -0.50 \\
\hline TDDFT/6-311(2+,2+) $\mathrm{G}^{* * \mathrm{i}}$ & 5.90 & 40.5 & 6.48 & 42.6 & -0.58 \\
\hline$\pi \mathrm{CASSCF} / 5 \mathrm{~s} 4 p 2 \mathrm{dR} / 3 \mathrm{~s} 2 p^{\mathrm{j}}$ & 6.48 & 67.8 & 6.58 & 24.4 & -0.10 \\
\hline$\sigma \pi \mathrm{RASSCF} / 5 \mathrm{~s} 4 p 2 \mathrm{dR} / 3 \mathrm{~s} 2 p^{\mathrm{j}}$ & 6.56 & 60.2 & 6.79 & 23.7 & -0.23 \\
\hline $\mathrm{IDDCI}+\mathrm{DC} / \mathrm{ANO}^{\mathrm{k}}$ & 5.88 & $\ldots$ & 6.57 & $\ldots$ & -0.69 \\
\hline Expt. & $6.25,{ }^{1} 5.92^{\mathrm{m}}$ & $\cdots$ & $\cdots$ & $\ldots$ & $\cdots$ \\
\hline
\end{tabular}

${ }^{\mathrm{a}}$ Reference 2 .

${ }^{\mathrm{b}}$ Reference 5 .

${ }^{\mathrm{c}}$ Reference 6.

${ }^{\mathrm{d}}$ Reference 51 .

${ }^{\mathrm{e}}$ Reference 3.

${ }^{\mathrm{f}}$ Reference 46.

${ }^{g}$ Reference 8.

\author{
${ }^{\mathrm{h}}$ Reference 9. \\ ${ }^{\mathrm{i}}$ Reference 10 \\ ${ }^{\mathrm{j}}$ Reference 56. \\ ${ }^{\mathrm{k}}$ Reference 53 . \\ ${ }^{\mathrm{l}}$ Reference 32. \\ ${ }^{\mathrm{m}}$ Reference 29.
}

trum has led to the suggestion that the vertical electronic energy difference is not necessarily equal to the energy corresponding to the intensity maximum. Rai and Buenker ${ }^{55}$ pointed out that it is expected the calculated vertical energy difference will be $0.2-0.3 \mathrm{eV}$ or even bigger compared to the energy corresponding to the intensity maximum.

Figure S3 shows the eight active orbitals for the $1 \mathrm{~B}_{\mathrm{u}}$ state at the ground-state geometry. ${ }^{21}$ As observed in cisbutadiene, the shape of the occupied $\pi^{*}$ orbital reveals the mixed valence and Rydberg character of that state at the CASSCF level. The expectation values $\left\langle z^{2}\right\rangle$ are collected in Table II. The value of $\left\langle z^{2}\right\rangle$ for $1 \mathrm{~B}_{\mathrm{u}}$ is computed to be 55.7 a.u. $^{2}$ at the $\operatorname{CASSCF}(4,8) / 6-31 \mathrm{G}^{*}+3 p$ level of calculation. This is more than twice the value for the $2 \mathrm{~A}_{\mathrm{g}}$ state $(23.8$ a.u. ${ }^{2}$ ) and somewhat higher than the value for the ionic state of the cis isomer (41.9 a.u. ${ }^{2}$ ). This suggests that the valenceRydberg mixing is larger in the trans isomer than it is in the cis. It is worth noting that CASSCF calculations of Cave ${ }^{56}$ give similar transition energies compare to our results but produce a more diffuse $1 \mathrm{~B}_{\mathrm{u}}$ state $\left(\left\langle z^{2}\right\rangle=67.8\right.$ a.u. $\left.^{2}\right)$.

In summary, $\mathrm{CASSCF}(4,8) / 6-31 \mathrm{G}^{*}+3 p$ vertical excitation energies place the $1 \mathrm{~B}_{\mathrm{u}}$ state slightly lower than the $2 \mathrm{~A}_{\mathrm{g}}$ state at the ground-state equilibrium geometry. This ordering is consistent with all the previous accurate calculations and experimental observations. We estimate the vertical transition energy of $1 \mathrm{~B}_{\mathrm{u}}$ to be about $0.3 \mathrm{eV}$ too high at the CASSCF level and the $1 \mathrm{~B}_{\mathrm{u}}-2 \mathrm{~A}_{\mathrm{g}}$ energy gap to be too small. The following calculations aim to improve these vertical excitation energies by recovering the most important missing part of the dynamic correlation with the use of a RASSCF wavefunction.

By using a double active space $(4,8)$ at the CASSCF level and an extended basis set in the $\pi$ orbital space, we have confidence that we can describe properly the fraction of the dynamic electron correlation that arises from the $\pi-\pi^{*}$ excitations. However CASSCF does not include important differential dynamic correlation effects. One might expect the dynamic correlation of the $\sigma$ electrons (i.e., double excitations of the $\sigma$ electrons) to be approximately the same for ionic and covalent states. However, polarization of the $\sigma$ electrons and semi-internal correlation (described by configurations involving simultaneous $\sigma-\sigma^{*}$ and $\pi-\pi^{*}$ excitations, inactive-virtual and active-active double excitations in general) depends strongly on the electronic character and is crucial for the description of ionic states. ${ }^{2}$ These dynamic polarization effects are included in what Cave and Davidson call $\sigma \pi$ correlation and it has been emphasized that the ionic $1 \mathrm{~B}_{\mathrm{u}}$ state of polyenes is much more sensitive to $\sigma \pi$ correlation than the covalent $2 \mathrm{~A}_{\mathrm{g}}$ state. $^{7,48}$

The effect of the $\sigma$ electrons is included in a RASSCF treatment as explained in Sec. II. The results are gathered in Table II. Compared to the CASSCF result, the vertical excitation energy of the $1 \mathrm{~B}_{\mathrm{u}}$ state is slightly improved when using RASSCF. There is little change in the diffuse nature of the state, which may be due to the limited subset of the possible $\sigma \rightarrow \sigma^{*}$ excitations included in the RASSCF calculation. On the other hand, a noticeable change in the vertical excitation energy to the $2 \mathrm{~A}_{\mathrm{g}}$ state is observed. This state is 

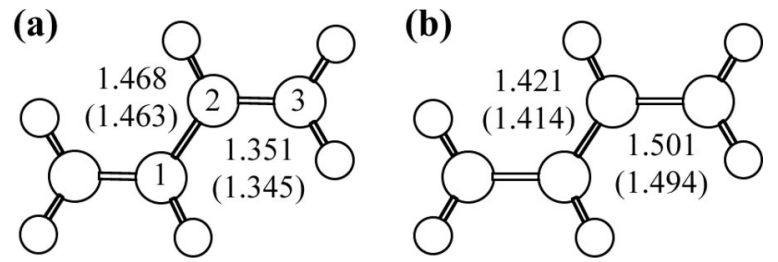

(c)

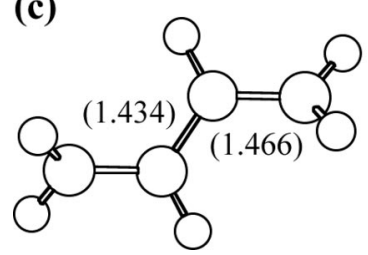

(d)

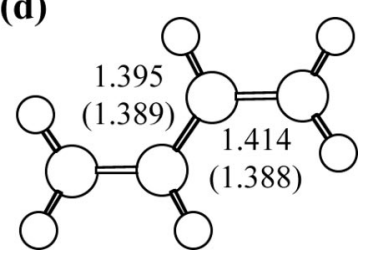

FIG. 2. $\operatorname{RASSCF}(22,9+5+12)[1,1] / 6-31 \mathrm{G}^{*}+3 p$ and $\operatorname{CASSCF}(4,8) / 6-$ $31 \mathrm{G}^{*}+3 p$ (in parentheses) optimized structures on the $1 \mathrm{~A}_{\mathrm{g}}, 2 \mathrm{~A}_{\mathrm{g}}$, and $1 \mathrm{~B}_{\mathrm{u}}$ potential energy surfaces of trans-butadiene. All bond lengths in $\AA$. (a) $1 \mathrm{~A}_{\mathrm{g}} C_{2 \mathrm{~h}}$ minimum, (b) $2 \mathrm{~A}_{\mathrm{g}} C_{2 \mathrm{~h}}$ maximum, (c) $2 \mathrm{~A}_{\mathrm{g}} C_{2}$ minimum, and (d) $1 \mathrm{~B}_{\mathrm{u}} C_{2 \mathrm{~h}}$ saddle point.

placed $6.99 \mathrm{eV}$ above the ground-state minimum at the RASSCF level, compared to the $6.59 \mathrm{eV}$ at the CASSCF level. Although this value seems too high, the energy gap between $1 \mathrm{~B}_{\mathrm{u}}$ and $2 \mathrm{~A}_{\mathrm{g}}(0.49 \mathrm{eV})$ is in very good agreement with the very accurate QR-CC3 calculations of Cronstrand et al. ${ }^{9}$ Allowing double excitations from RAS1 reduces the energy gap to $0.33 \mathrm{eV}$. Our RASSCF vertical transition energies also agree with the $\sigma \pi$ RASSCF calculations of Cave (for which no computational details are given). ${ }^{56}$ It is worth noting that our values are also in good agreement with the MRCI/MIDI3 results, ${ }^{5}$ which are uniformly higher than all other correlated results.

\section{Topology of the potential energy surfaces}

CASSCF $(4,4) / 6-31 \mathrm{G}^{*}, \quad \operatorname{CASSCF}(4,8) / 6-31 \mathrm{G}^{*}+3 p$, and $\operatorname{RASSCF}(22,9+5+12)[1,1] / 6-31 \mathrm{G}^{*}+3 p$ have been used to investigate the topology of the PESs of the first two

TABLE III. Optimized geometries of the three lowest singlet states of transbutadiene at the CASSCF and RASSCF level. CASSCF $(4,8) / 6-31 \mathrm{G}^{*}+3 p$ in normal print, $\operatorname{CASSCF}(4,4) / 6-31 \mathrm{G}^{*}$ in parentheses, $\operatorname{RASSCF}(22,9+5$ $+12)[1,1] / 6-31 \mathrm{G}^{*}+3 p$ in bold, and CASPT2 ${ }^{\mathrm{a}}$ in brackets.

\begin{tabular}{lccc}
\hline \hline \multicolumn{1}{c}{ Geometry } & $C_{1} C_{2}(\AA)$ & $C_{2} C_{3}(\AA)$ & $\angle C_{1} C_{2} C_{3}(\mathrm{deg})$ \\
\hline $1 \mathrm{~A}_{\mathrm{g}} C_{2 \mathrm{~h}}$ & 1.463 & 1.345 & 124.1 \\
& $(1.465)$ & $(1.344)$ & $(124.1)$ \\
& $\mathbf{1 . 4 6 8}$ & $\mathbf{1 . 3 5 1}$ & $\mathbf{1 2 3 . 9}$ \\
& {$[1.454]$} & {$[1.348]$} & {$[123.6]$} \\
$2 \mathrm{~A}_{\mathrm{g}} C_{2 \mathrm{~h}}$ & 1.414 & 1.494 & 123.0 \\
& $(1.418)$ & $(1.499)$ & $(123.4)$ \\
& $\mathbf{1 . 4 2 1}$ & $\mathbf{1 . 5 0 1}$ & $\mathbf{1 2 3 . 0}$ \\
& {$[1.401]$} & {$[1.500]$} & {$[123.3]$} \\
$2 \mathrm{~A}_{\mathrm{g}} C_{2}$ & 1.434 & 1.466 & 122.9 \\
& $(1.444)$ & $(1.468)$ & $(122.8)$ \\
$1 \mathrm{~B}_{\mathrm{u}} C_{2 \mathrm{~h}}$ & 1.389 & 1.388 & 121.8 \\
& $(1.397)$ & $(1.411)$ & $(125.9)$ \\
& $\mathbf{1 . 3 9 5}$ & $\mathbf{1 . 4 1 4}$ & $\mathbf{1 2 2 . 2}$ \\
& {$[1.399]$} & {$[1.421]$} & {$[124.1]$} \\
$1 \mathrm{~B}_{\mathrm{u}} / 2 \mathrm{~A}_{\mathrm{g}} \mathrm{CI} C_{2 \mathrm{~h}}$ & 1.392 & 1.347 & 126.4 \\
& $\mathbf{1 . 4 1 1}$ & $\mathbf{1 . 4 1 8}$ & $\mathbf{1 2 4 . 5}$ \\
\hline \hline
\end{tabular}

${ }^{\mathrm{a}}$ Reference 11 .

TABLE IV. Nonvertical excitation energies of the $1 \mathrm{~B}_{\mathrm{u}}$ and $2 \mathrm{~A}_{\mathrm{g}}$ states of trans-butadiene. Geometries in Table III and Fig. 2.

\begin{tabular}{|c|c|c|c|c|}
\hline Method & $\begin{array}{l}1 \mathrm{~B}_{\mathrm{u}} C_{2 \mathrm{~h}} \\
\Delta E(\mathrm{eV})\end{array}$ & $\begin{array}{l}2 \mathrm{~A}_{\mathrm{g}} C_{2 \mathrm{~h}} \\
\Delta E(\mathrm{eV})\end{array}$ & $\begin{array}{r}2 \mathrm{~A}_{\mathrm{g}} C_{2} \\
\Delta E(\mathrm{eV})\end{array}$ & $\begin{array}{r}2 \mathrm{~A}_{\mathrm{g}} C_{\mathrm{i}} \\
\Delta E(\mathrm{eV})\end{array}$ \\
\hline $\operatorname{CASSCF}(4,4) / 6-31 \mathrm{G}^{*}$ & 8.29 & 5.51 & 5.30 & 5.32 \\
\hline $\operatorname{CASSCF}(4,8) / 6-31 \mathrm{G}^{*}+3 p$ & 6.32 & 5.48 & 5.27 & $\cdots$ \\
\hline $\begin{array}{l}\operatorname{RASSCF}(22,9+5+12) \\
{[1,1] / 6-31 \mathrm{G}^{*}+3 p}\end{array}$ & 6.22 & 5.81 & $\cdots$ & $\cdots$ \\
\hline $\begin{array}{l}\operatorname{CASSCF}(4,5) / \mathrm{MIDI} 3 \\
\quad+2 p d^{\mathrm{a}}\end{array}$ & 6.36 & 5.53 & 5.26 & 5.28 \\
\hline $\mathrm{MRCI} / \mathrm{MIDI} 3+2 p d^{\mathrm{a}}$ & 6.44 & 5.57 & 5.26 & 5.29 \\
\hline$\sigma \pi-\mathrm{CI} / \mathrm{ANO}^{\mathrm{b}}$ & 5.74 & 5.66 & $\cdots$ & $\cdots$ \\
\hline TD-CC/MIDI3 $+2 p^{\mathrm{c}}$ & 6.04 & $\cdots$ & $\cdots$ & $\cdots$ \\
\hline $\mathrm{MRMP} / \mathrm{QZ} 3 p^{\mathrm{d}}$ & 5.93 & 5.49 & $\ldots$ & $\cdots$ \\
\hline CASPT2/ANO & $\ldots$ & 5.16 & 5.02 & $\ldots$ \\
\hline CASPT2/6-31G*f & 6.18 & 5.37 & $\ldots$ & $\cdots$ \\
\hline Expt. & $\begin{array}{c}5.73,{ }^{\mathrm{g}} \\
5.92-6.02^{\mathrm{h}}\end{array}$ & & & \\
\hline
\end{tabular}

${ }^{\mathrm{a}}$ Reference 5 .

${ }^{\mathrm{b}}$ Reference 48.

${ }^{\mathrm{c}}$ Reference 57.

${ }^{\mathrm{d}}$ Reference 3.

${ }^{\mathrm{e}}$ Reference 39.
${ }^{\mathrm{f}}$ Reference 11.
${ }^{\mathrm{g}}$ Reference 31.
${ }^{\mathrm{h}}$ Reference 25.

excited states of trans-butadiene. Structural information is collected in Fig. 2 and Table III. Relative energies are collected in Table IV.

Geometry optimizations on the $2 \mathrm{~A}_{\mathrm{g}}$ PES were performed with $C_{2 \mathrm{~h}}, C_{2}$, and $C_{\mathrm{i}}$ symmetry constraints. Three stationary points were found, but only the $C_{2}$ structure was found to be a minimum. Indeed, a frequency analysis showed that the planar $C_{2 \mathrm{~h}}$ structure has four negative directions of curvature, as was also found by Zerbetto and Zgierski ${ }^{40}$ and Lappe and Cave. ${ }^{39}$ The $C_{\mathrm{i}}$ structure is found to be a transition state. Hence the $C_{2}$ minimum is the lowest-energy point on the $2 \mathrm{~A}_{\mathrm{g}}$ PES and lies about $5 \mathrm{kcal} \mathrm{mol}^{-1}$ below the $C_{2 \mathrm{~h}}$ structure, in good agreement with previous MCSCF and MRCI results. This minimum is located $5.27 \mathrm{eV}$ above the groundstate minimum, in very good agreement with the MRCI result, but $0.25 \mathrm{eV}$ too high compared to the CASPT2 calculations of Lappe and Cave. ${ }^{39}$ The planar $C_{2 \mathrm{~h}}$ structure lies $5.48 \mathrm{eV}$ above that minimum at the CASSCF level. This is $0.1 \mathrm{eV}$ below the MRCI result of Szalay et al. ${ }^{5}$ and $0.11 \mathrm{eV}$ above the CASPT2 result of Page and Olivucci, ${ }^{11}$ but in very good agreement with the MRMP result of Nakayama et al. ${ }^{3}$ On the other hand, the RASSCF nonvertical excitation energy $(5.81 \mathrm{eV})$ is closer to the MRCI value, but seems too high as observed in the previous subsection. Notice that Lappe and Cave ${ }^{39}$ propose a much lower transition energy at the CASPT2 level.

We optimized a $C_{2 \mathrm{~h}}$ stationary point on the $1 \mathrm{~B}_{\mathrm{u}}$ PES. The nature of this stationary point depends on the level of calculation used: $\operatorname{CASSCF}(4,4) / 6-31 \mathrm{G}^{*}$ and CASSCF $(4,4) / 6-31+\mathrm{G}^{*}$ frequency calculations produce two and one imaginary frequencies respectively, whereas CASSCF $(4,8) / 6-31+\mathrm{G}^{*}$ produces none. Problems occur when computing the $\operatorname{CASSCF}(4,8) / 6-31 \mathrm{G}^{*}+3 p$ frequencies (as discussed in the Computational Methods section). In any case this planar $C_{2 \mathrm{~h}}$ structure is not a global minimum on the $1 \mathrm{~B}_{\mathrm{u}}$ PES. Indeed, it has been shown that the minimum on the ionic PES is nonplanar. Rai and Buenker ${ }^{55}$ have re- 
ported a minimum at a geometry in which all $\mathrm{C}-\mathrm{C}$ bond lengths are equal and both terminal $\mathrm{CH}_{2}$ groups are rotated by $10^{\circ}$. Nonetheless, the planar $C_{2 \mathrm{~h}}$ structure is of interest to examine the energetic effects of significant changes in the bond lengths in this excited state. $\operatorname{CASSCF}(4,8) / 6-31 \mathrm{G}^{*}$ $+3 p$ places this state $6.32 \mathrm{eV}$ above the ground-state minimum, in fairly good agreement with the MRCI value. ${ }^{5}$ The nonvertical excitation energy is lowered by $0.1 \mathrm{eV}$ at the RASSCF level, which gives a better agreement with the MRMP and time-dependent coupled-cluster (TD-CC) results (Table IV). This is also in very good agreement with the CASPT2 calculations of Page and Olivucci, ${ }^{11}$ which place that state $6.18 \mathrm{eV}$ above the ground-state minimum. The nonvertical excitation energy to the $1 \mathrm{~B}_{\mathrm{u}}$ state has been measured to be $5.73 \mathrm{eV}$ (Ref. 31) and 5.92-6.02 eV (Ref. 25). This is substantially lower than our RASSCF result. Nonetheless, the $C_{2 \mathrm{~h}}$ structure is not a minimum and stabilization upon rotation of a $\mathrm{CH}_{2}$ group or pyramidalization is expected for that state. It is worth noting as well that the effect of the $\sigma \pi$ correlation on these geometries is rather small at the MCSCF level. The RASSCF planar geometry for the $1 \mathrm{~B}_{\mathrm{u}}$ state is in fairly good agreement with the TD-CC optimized geometry of Szalay and Bartlett. ${ }^{57}$ The central and terminal $\mathrm{C}-\mathrm{C}$ bond lengths agree to within 0.001 and $0.01 \AA$, respectively.

Finally, we located a low-lying conical intersection between the $1 \mathrm{~B}_{\mathrm{u}}$ and $2 \mathrm{~A}_{\mathrm{g}}$ states. This critical feature on the PESs accounts for the observed ultrafast radiationless decay of the optically bright $1 \mathrm{~B}_{\mathrm{u}}$ state into the dark $2 \mathrm{~A}_{\mathrm{g}}$ state. ${ }^{36}$ Ostojic and Domcke ${ }^{51}$ investigated these two PESs at the CASPT2 level. They identified coordinates along which such a crossing is observed, but no optimized geometry was proposed by these authors or by Page and Olivucci. ${ }^{11}$ To our knowledge, this is the first time that such a structure is reported, based on full optimization of the geometrical parameters at an accurate $a b$ initio level. The crossing geometry has been optimized constraining a planar configuration of $C_{2 \mathrm{~h}}$ symmetry at the $\operatorname{CASSCF}(4,8) / 6-31 \mathrm{G}^{*}+3 p$ and $\operatorname{RASSCF}(22,9+5+12)[1,1] / 6-31 \mathrm{G}^{*}+3 p$ levels using state-averaged orbitals between the two states of interest. This is because ultrafast electronic relaxation pathway on the ionic state is not expected to involve twisting of the CC bonds. ${ }^{36}$ The structure is displayed in Fig. 3 along with the two directions that lift the degeneracy-i.e., the derivative coupling (DC) and gradient difference (GD) vectors. This conical intersection is located $14 \mathrm{kcal} \mathrm{mol}^{-1}$ above the $1 \mathrm{~B}_{\mathrm{u}} C_{2 \mathrm{~h}}$ structure at the CASSCF level and about 4 $\mathrm{kcal} \mathrm{mol}^{-1}$ above at the RASSCF level. The central CC bond has a similar length to the $1 \mathrm{~B}_{\mathrm{u}} C_{2 \mathrm{~h}}$ structure, whereas the terminal CC bond is $0.04 \AA$ shorter at the CI structure. The structure obtained at the RASSCF level is dramatically different. Indeed, the central CC bond becomes longer by 0.02 $\AA$ compared to CASSCF, whereas the terminal CC bond is stretched by $0.07 \AA$. As a result, the $1 \mathrm{~B} / 2 \mathrm{~A}$ CI structure is much closer to the $1 \mathrm{~B}_{\mathrm{u}}$ optimized structure at the RASSCF level. This is in much better agreement with the CASPT2 results obtained by Page and Olivucci for the cis isomer, where they found a planar $C_{2 \mathrm{v}}$ CI close to the $1 \mathrm{~B}_{2}$ minimum. ${ }^{11}$ Therefore, we can safely conclude that RASSCF gives much better ionic/covalent CI structures than CASSCF.

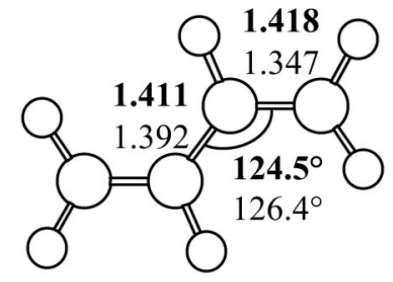

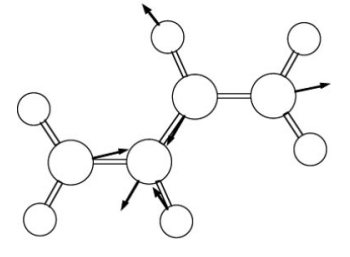

CASSCF DC vector

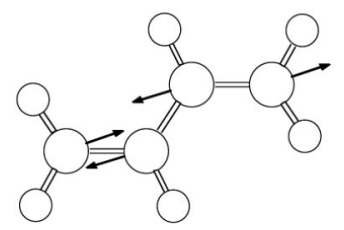

RASSCF DC vector

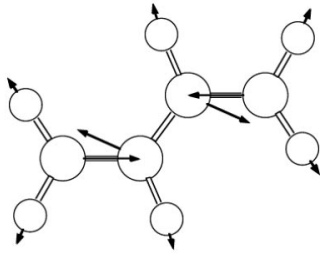

CASSCF GD vector

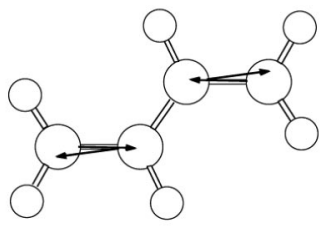

RASSCF GD vector
FIG. 3. $\operatorname{CASSCF}(4,8) / 6-31 \mathrm{G}^{*}+3 p$ and $\operatorname{RASSCF}(22,9+5+12)[1,1] /$ $6-31 \mathrm{G}^{*}+3 p$ (in bold) optimized $1 \mathrm{~B}_{\mathrm{u}} / 2 \mathrm{~A}_{\mathrm{g}}$ conical intersection in transbutadiene. All bond lengths in $\AA$. The derivative coupling (DC) and gradient difference (GD) vectors are also shown. The CASSCF and RASSCF stateaveraged energies of $1 \mathrm{~B}_{\mathrm{u}}$ and $2 \mathrm{~A}_{\mathrm{g}}$ are $-154.72752 E_{\mathrm{h}},-154.72700 E_{\mathrm{h}}$ and $-154.82701 E_{\mathrm{h}},-154.82683 E_{\mathrm{h}}$, respectively.

This is due to the fact that the geometry of such structures is very sensitive to the differential dynamic correlation between the two states, which is describe in a more balanced way at the RASSCF level.

\section{Hexatriene}

Aside from a shift of all transitions to lower energy, the spectroscopy of hexatriene is quite similar to that of butadiene and the subject of a large number of experimental studies. ${ }^{58-72}$ The dominant feature in the spectrum is a broad, intense peak attributed to the $1 \mathrm{~B}_{2}$ and $1 \mathrm{~B}_{\mathrm{u}} \pi \rightarrow \pi^{*}$ transition for the $c Z c$ and $t E t$ isomers, respectively, followed by a series of sharp Rydberg peaks. These isomers have been invoked in the wide discussion about the order of the two lowest valence states that has plagued the spectroscopy of linear polyenes. ${ }^{31}$ No evidence of the $2 \mathrm{~A}$ valence state under the lowest optically allowed 1B state has been found in earlier studies, as in butadiene. ${ }^{31}$ Because of the size of hexatriene, fewer extensive ab initio calculations have been performed on it. On the other hand, hexatriene seemingly offers less difficulty than butadiene, because of a decrease in the Rydberg-valence mixing, which was so crucial for the lower states of butadiene. Most of the calculations performed were focused on determining the nature of the lowest-lying excited states and their ordering. ${ }^{1-3,7,9,10,52,73,74}$ In particular, the order between the $2 \mathrm{~A}$ and $1 \mathrm{~B}$ states at the ground-state geometry is still an open question. Whereas the $1 \mathrm{~B}$ state was unequivocally lower in energy than the $2 \mathrm{~A}$ state for butadiene, the results are divided for hexatriene. The inflection 
point for the vertical excitation energies between the onephoton allowed 1B state and the two-photon 2A state in the series of linear polyenes is likely to occur for this system. ${ }^{9}$

\section{1. cZc-hexatriene}

The most intense peak observed in optical absorption spectra was obtained at $4.92 \mathrm{eV}^{59,63,75}$ Electron impact spectroscopy has also been used, but could not distinguish between isomers of hexatriene because of insufficient resolution. ${ }^{60-63}$ Multiphoton ionization ${ }^{64}$ and two-photon absorption ${ }^{65}$ spectroscopy focused mainly on the $t E t$ isomer. Relatively few calculations have been performed on $c Z c$-hexatriene: to our knowledge, the first ab initio calculations reported for the excited states of the $c Z c$ isomer are the work of Serrano-Andrés et al. in 1994. ${ }^{1}$ These authors placed the ionic $1 \mathrm{~B}_{2}$ state just $0.04 \mathrm{eV}$ below the $2 \mathrm{~A}_{1}$ state at the ground-state planar geometry using CASPT2. Recently, Woywod and co-workers ${ }^{74}$ have recomputed the vertical excitation energies at the CASPT2 level and concluded that the $2 \mathrm{~A}_{1}$ state is at most $0.5 \mathrm{eV}$ and possibly not more than 0.1 $\mathrm{eV}$ below the $1 \mathrm{~B}_{2}$ state, which disagrees with the previous results of Serrano-Andrés et al. Hence it is not clear whether the ionic state is below or above the covalent state: the two low-lying excited states of $c Z c$-hexatriene are in the same energy region at the ground state geometry.

Table S4 collects our vertical excitation energies at the planar ground-state geometry along with the other results mentioned above. ${ }^{21}$ CASSCF $(6,6) / 6-31 G^{*}$ results are poor, since the ionic state is placed $1.5 \mathrm{eV}$ above the covalent state (with state-averaged orbitals) at this level. [The use of stateaveraged orbitals is purely due to technical reasons. The wrong state order between $1 \mathrm{~B}_{2}$ and $2 \mathrm{~B}_{2}$ is obtained when using the incomplete $6-31 \mathrm{G}^{*}$ basis set and $\mathrm{CAS}(6,6)$. Therefore, the computation of the $1 \mathrm{~B}_{2}$ state is not possible without state averaging as the $2 \mathrm{~B}_{2}$ state is an intruder state at this level of calculation. This issue disappears when using our $6-31 \mathrm{G}^{*}+3 p$ basis set with a larger active space, as the valence ionic $1 \mathrm{~B}_{2}$ state is then lower than $2 \mathrm{~B}_{2}$ as observed experimentally.] Doubling the active space and explicitly including $3 p$ functions in the basis set reduces the energy gap to $0.7 \mathrm{eV}$. This result is somewhat disappointing since we obtained the right order of the states at this level of calculation for butadiene. This is due to a lower valence-Rydberg mixing character for the ionic state of hexatriene $\left(\left\langle x^{2}\right\rangle\right.$ $=34.8$ a.u. $\left.{ }^{2}\right)$. Hence the stabilization effect of $3 \pi$ orbitals is less pronounced. Nonetheless, the $1 \mathrm{~B}_{2}$ state can now be calculated without state averaging. Besides, our CASSCF result is in better agreement with other high-level calculations than the $\operatorname{CASSCF}(6,10)$ result of Serrano-Andrés and co-workers. ${ }^{1}$ The use of diffuse functions in the ANO basis set seems to be responsible for a stabilization of Rydberg states to the detriment of the valence $1 \mathrm{~B}_{2}$ state, as observed for butadiene. The most important result is that RASSCF calculations improve substantially our CASSCF result, as the two states becomes very close at this level. Indeed, the $2 \mathrm{~A}_{1}$ state is placed $0.14 \mathrm{eV}$ below the $1 \mathrm{~B}_{2}$ state and only $0.01 \mathrm{eV}$ below when using state-averaged orbitals.

CASSCF and RASSCF levels of theory have been used to investigate the topology of the PESs of $c Z c$-hexatriene.
Structural information is collected in Fig. S4 and Table S5. Relative energies are collected in Table $\mathrm{S}^{2{ }^{21}} \mathrm{~A}$ $\operatorname{CASSCF}(6,6)$ frequency analysis of the optimized $C_{2 v}$ planar structure on the ground state reveals two imaginary frequencies. Two nonplanar structures with $C_{2}$ and $C_{\mathrm{s}}$ symmetry were found, 6.4 and $5.2 \mathrm{kcal} \mathrm{mol}^{-1}$, respectively, below the planar structure. The $C_{2}$ structure is a minimum whereas the $C_{\mathrm{s}}$ one displays one imaginary frequency. Most of the theoretical studies reported in the literature ${ }^{1,74,76}$ used a $C_{2 \mathrm{v}}$ equilibrium geometry for the ground state of cis-hexatriene in agreement with the interpretation of experimental observations in Ref. 77. On the other hand, electron diffraction data $^{78}$ and resonance Raman spectroscopy ${ }^{79}$ suggested a nonplanar $S_{0}$ minimum structure in agreement with our CASSCF calculations and the calculations of Sakai and Takane. ${ }^{80}$

$C_{2 \mathrm{v}}$ and $C_{2}$ structures were optimized on the first two excited states of $c Z c$-hexatriene. As observed in butadiene, the differences in bond lengths between $\operatorname{CASSCF}(6,6) /$ 6-31G* and $\operatorname{CASSCF}(6,12) / 6-31 \mathrm{G}^{*}+3 p$ optimized structures are very small. The planar $1 \mathrm{~B}_{2}$ structure was optimized using state-averaged $\operatorname{CASSCF}(6,6) / 6-31 \mathrm{G}^{*}$ because of the nearby $2 \mathrm{~B}_{2}$ state in the region of the $1 \mathrm{~B}_{2}$ minimum. Only the $C_{2}$ structure for the $1 \mathrm{~B}_{2}$ state was found to be a minimum. As shown in Table S6, the nonvertical transition energies are substantially lowered for the ionic state when using $\operatorname{CASSCF}(6,12) / 6-31 \mathrm{G}^{*}+3 p$ : a stabilization of 1.0 and 0.7 $\mathrm{eV}$ is observed for the $C_{2 \mathrm{v}}$ and $C_{2}$ structures, respectively. Unfortunately, no other theoretical data are available in the literature concerning the nonvertical excitation energies in this system.

$\operatorname{RASSCF}(32,13+6+19)[1,1] / 6-31 \mathrm{G}^{*}+3 p$ single-point calculations have been performed for the two $\mathrm{A}_{1}$ states at the CASSCF optimized geometries. Geometry optimization of the planar $1 \mathrm{~B}_{2}$ state was performed as well (see Table S5). As for butadiene, substantial changes in the bond lengths are observed compared to the CASSCF geometry. Moreover, the $1 \mathrm{~B}_{2}$ state is further stabilized by $0.6 \mathrm{eV}$ compared to the best CASSCF calculation (see Table S6). Based on these results, we predict the $2 \mathrm{~A}_{1}$ state to be the lowest excited state in the $0-0$ sense. It is worth noting that the two excited states are nearly degenerate at the Franck-Condon geometry, and therefore a $1 \mathrm{~B} / 2 \mathrm{~A}$ conical intersection is located in its near vicinity. Therefore, we expect fast radiationless decay to occur from the ionic state to the dark $2 \mathrm{~A}_{\mathrm{g}}$ state soon after excitation from the Franck-Condon region.

\section{2. tEt-hexatriene}

The $1 \mathrm{~B}_{\mathrm{u}}$ state of $t E t$-hexatriene is well characterized experimentally. The maximum intensity occurs at $4.93 \mathrm{eV}$ (Ref. 59) or $4.95 \mathrm{eV}$ (Ref. 63), whereas the electron-impact intensity maximum appears at $5.13 \mathrm{eV}$ (Ref. 61), which is considered to be the vertical transition energy. A two-photon absorption study of hexatriene finds an allowed band with a maximum at $5.21 \mathrm{eV}$ (Ref. 71), which corresponds to the vertical transition energy to the $2 \mathrm{~A}_{\mathrm{g}}$ state. Extensive $a b$ initio calculations have been performed on $t E t$-hexatriene, but as for the cis isomer, it is still debated whether the $1 \mathrm{~B}_{\mathrm{u}}$ or $2 \mathrm{~A}_{\mathrm{g}}$ state is the lowest one. Most of the accurate calculations 
place the two states very close in energy (within $0.2 \mathrm{eV}$ ) at the ground-state geometry. The only exception is the CASPT2 calculations of Woywod et al., ${ }^{73}$ which place the $1 \mathrm{~B}_{\mathrm{u}}$ state $0.5 \mathrm{eV}$ above the $2 \mathrm{~A}_{\mathrm{g}}$ state. As for $c Z c$-hexatriene, it is likely that the two states are nearly degenerate at the ground-state geometry. The reaction path involving a threestate photochemical pathway has also been documented. ${ }^{81}$

Table S7 collects our CASSCF vertical excitation energies at the ground-state geometry, along with the most recent calculations available in the literature. ${ }^{21}$ As expected, $\operatorname{CASSCF}(6,6) / 6-31 \mathrm{G}^{*}$ gives poor results: the ionic $1 \mathrm{~B}_{\mathrm{u}}$ state is placed $1.9 \mathrm{eV}$ above the $2 \mathrm{~A}_{\mathrm{g}}$ state. As for the cis isomer, state averaging with the valence $2 \mathrm{~B}_{\mathrm{u}}$ state was necessary to avoid mixing with this state. Doubling the active space and explicitly including $3 p$ functions in the basis set reduces the energy gap to $0.65 \mathrm{eV}$, in much better agreement with experiment than the CASSCF(6,8)/ANO result of Serrano-Andres et al. $^{2}$ These results are very similar to those obtained for the $c Z c$ isomer. RASSCF calculations improve this result, as the two states become closer in energy. Indeed, the $2 \mathrm{~A}_{\mathrm{g}}$ state is placed $0.28 \mathrm{eV}$ below the $1 \mathrm{~B}_{\mathrm{u}}$ state when using stateaveraged orbitals. This result is in reasonable agreement with previous theoretical calculations, although the excitation energies are probably slightly too high.

CASSCF and RASSCF were used to investigate the topology of the PESs considered. Structural information is collected in Fig. S5 and Table S8. Relative energies are collected in Table S9. ${ }^{21}$ The ground-state equilibrium geometry is found to be a planar $C_{2 \mathrm{~h}}$ structure, in agreement with all previous calculations and experimental observations. Planar $C_{2 \mathrm{~h}}$ structures were optimized on the $1 \mathrm{~B}_{\mathrm{u}}$ and $2 \mathrm{~A}_{\mathrm{g}}$ PESs. In the case of the $2 \mathrm{~A}_{\mathrm{g}}$ state, two imaginary frequencies characterized the planar structure and a lower-energy nonplanar $C_{2}$ minimum was located. This $C_{2}$ minimum is only $0.03 \mathrm{kcal} \mathrm{mol}^{-1}$ below the $C_{2 \mathrm{~h}}$ structure at the CASSCF $(6,6) / 6-31 \mathrm{G}^{*}$ level. The carbon skeleton is almost identical to the $C_{2 \mathrm{~h}}$ structure: only one of the two terminal hydrogens is out of the molecular plane by $14^{\circ}$. Considering the quasidegeneracy between the $C_{2}$ and the $C_{2 \mathrm{~h}}$ structures as well as the closeness of those two structures, the topology of the PES may change with the level of calculation. Therefore, based on this result, we cannot say whether the $2 \mathrm{Ag}_{\mathrm{g}}$ minimum is planar or not. Cave and Davidson report inplane relaxation effects of $1.22 \mathrm{eV},{ }^{48}$ in very good agreement with the $1.18 \mathrm{eV}$ obtained at the RASSCF level. This relaxation effect is less pronounced at the MRMP level, with a stabilization of $0.92 \mathrm{eV}^{3}$ The RASSCF nonvertical excitation energy to $2 \mathrm{~A}_{\mathrm{g}}$ is in good agreement with the QR-CC estimation of Cronstrand et al. ${ }^{9}$ as well.

In the case of the ionic state, the $\operatorname{CASSCF}(6,6) / 6-31 \mathrm{G}^{*}$ frequency calculation using state-averaged orbitals suggests that the $C_{2 \mathrm{~h}}$ structure is a minimum. This has to be taken with caution as well, since we have shown in the case of butadiene that the frequency analysis for the ionic state is dependent on the level of theory used, especially the basis set. As shown in Table S9, the nonvertical transition energy is considerably improved for the ionic state when using $\operatorname{CASSCF}(6,12) / 6-31 \mathrm{G}^{*}+3 p$. A stabilization of $1.14 \mathrm{eV}$ is obtained compared to the $\operatorname{CASSCF}(6,6)$ calculation.
$\operatorname{RASSCF}(32,13+6+19)[1,1] / 6-31 \mathrm{G}^{*}+3 p \quad$ single-point calculations of the two $\mathrm{A}_{\mathrm{g}}$ states at CASSCF optimized geometries and geometry optimization of the planar $1 \mathrm{~B}_{\mathrm{u}}$ state have been performed as well. As observed previously, the bond lengths are stretched at the RASSCF level compared to the CASSCF geometry. Moreover, the $1 \mathrm{~B}_{\mathrm{u}}$ state is further stabilized by $0.5 \mathrm{eV}$ compared to the best CASSCF calculation. This result is $0.8 \mathrm{eV}$ higher than the MRMP calculation of Nakayama et al. ${ }^{3}$ and the QR-CC estimation of Cronstrand et al. ${ }^{9}$ Nonetheless, the RASSCF in-plane relaxation effect of $0.39 \mathrm{eV}$ is in good agreement with the relaxation energy of $0.46 \mathrm{eV}$ proposed by Cave and Davidson. ${ }^{48}$ As for the $2 \mathrm{~A}_{\mathrm{g}}$ state, this relaxation effect is less pronounced at the MRMP level with a stabilization of $0.26 \mathrm{eV}$. The nonvertical excitation energies suggest that the $2 \mathrm{~A}_{\mathrm{g}}$ state is the lowest excited state in the $0-0$ sense. As for the $c Z c$ isomer, it is expected that a fast radiationless decay occurs from the ionic state to the dark $2 \mathrm{~A}_{\mathrm{g}}$ state soon after excitation from the Franck-Condon region because of the proximity of those states, in agreement with experimental observations ${ }^{82}$ and previous correlated calculations. ${ }^{81}$

\section{CONCLUSION}

In this paper, we have shown how CASSCF-based $a b$ initio methods can be used to investigate potential energy surfaces of systems for which a balanced treatment of the dynamic correlation is required. The model systems investigated were the polyenes butadiene and hexatriene, which are well known for the challenging theoretical problems they offer. Indeed, these systems present two low-lying excited states involving a different valence-Rydberg character, a singly and doubly excited nature, with covalent or ionic (in the valence bond sense) character. Therefore, the dynamic correlation plays a crucial role in the description of these electronic states. Moreover, these two states are very close in energy at the ground-state geometry and their energetic order is still very much debated.

As shown in previous studies, the use of an enlarged $\pi$ active space is a crucial first step towards recovering part of the dynamic correlation and, consequently, obtaining the proper excited-state ordering at the ground-state geometry. On top of this, we have built a new conventional oneelectron basis set by adding optimized explicit $3 p$ basis functions on the carbon centers to the standard $6-31 \mathrm{G}^{*}$ basis set in order to improve the description of the ionic 1B states. This original yet simple technique proved to be more efficient to recover part of the $\pi \pi^{*}$ dynamic correlation than using the standard $6-31+\mathrm{G}^{*}$ basis set. This can be explained by the fact that the 1B state is a valence ionic state, but not a Rydberg state, and $3 p$ functions are therefore more relevant than simple diffuse Rydberg $p$ functions. The CASSCF results on the vertical excitation energy of the $1 \mathrm{~B}_{2}$ state of cis-butadiene showed that the $6-31 \mathrm{G}^{*}+3 p$ basis set is as accurate as the extended aug-cc-pVDZ basis set. Hence we have found a way of building an effective basis set to describe valence ionic states without using too many basis functions. Moreover, this basis set is built in such a way that 
the integral code of GAUSSIAN (Ref. 17) still runs very efficiently.

The CASSCF $(4,8) / 6-31 \mathrm{G}^{*}+3 p$ level of calculation gives qualitative results for butadiene, but fails to describe accurately the ionic/covalent conical intersection geometries. This approach does not provide the proper topology of the excited PESs for hexatriene in the Franck-Condon region either. The inclusion of $\sigma \pi$ correlation was shown to be necessary, and RASSCF has proved to be a successful approach to achieve this. The valence-core correlation energy is recovered by adding single $\sigma$ excitations to all the $\pi$ configurations. This gives a balanced description of the differential electron correlation and greatly improved results for the topology of PESs in hexatriene.

Finally, we emphasize that RASSCF has several advantages over correlated methods like CASPT2 or MRCI. Indeed, those methods do not have analytical energy gradients available, ${ }^{83}$ which means they have to be computed numerically. The computation of the first derivatives of the energy rapidly becomes unfeasible as the number of atoms increases. Hence such methods are presently limited for studying global PESs of chemical systems. On the other hand, RASSCF can approach the accuracy of methods like CASPT2 and MRCI, as we have shown in this study, and enables full geometry optimizations to be performed as analytical energy gradients are available. With such an approach, the calculation of relaxation paths and the simulation of the dynamics of the photochemical processes that take place in butadiene and hexatriene can be considered.

${ }^{1}$ L. Serrano-Andrés, B. O. Roos, and M. Merchán, Theor. Chim. Acta 87, 387 (1994).

${ }^{2}$ L. Serrano-Andrés, M. Merchán, I. Nebot-Gil, R. Lindh, and B. O. Roos, J. Chem. Phys. 98, 3151 (1993).

${ }^{3}$ K. Nakayama, H. Nakano, and K. Hirao, Int. J. Quantum Chem. 66, 157 (1998).

${ }^{4}$ P. G. Szalay, A. Karpfen, and H. Lischka, Chem. Phys. 141, 355 (1990).

${ }^{5}$ P. G. Szalay, A. Karpfen, and H. Lischka, Chem. Phys. 130, 219 (1989).

${ }^{6}$ R. J. Cave and E. R. Davidson, J. Phys. Chem. 91, 4481 (1987)

${ }^{7}$ R. J. Cave and E. R. Davidson, J. Phys. Chem. 92, 614 (1988).

${ }^{8}$ J. D. Watts, S. R. Gwaltney, and R. J. Bartlett, J. Chem. Phys. 105, 6979 (1996).

${ }^{9}$ P. Cronstrand, O. Christiansen, P. Norman, and H. Ågren, Phys. Chem. Chem. Phys. 3, 2567 (2001).

${ }^{10}$ C.-P. Hsu, S. Hirata, and M. Head-Gordon, J. Phys. Chem. A 105, 451 (2001).

${ }^{11}$ C. S. Page and M. Olivucci, J. Comput. Chem. 24, 298 (2003); (private communication).

${ }^{12}$ J. Olsen, B. O. Roos, P. Jørgensen, and H. J. A. Jensen, J. Chem. Phys. 89, 2185 (1988).

${ }^{13}$ P.-A. Malmqvist, A. Rendell, and B. O. Roos, J. Phys. Chem. 94, 5477 (1990).

${ }^{14}$ W. T. Borden and E. R. Davidson, Acc. Chem. Res. 29, 67 (1996).

${ }^{15}$ R. Ditchfield, W. J. Hehre, and J. A. Pople, J. Chem. Phys. 56, 2257 (1972); P. C. Hariharan and J. A. Pople, Theor. Chim. Acta 28, 213 (1973); T. Clark, J. Chandrasekhar, G. W. Spitznagel, and P. V. Schleyer, J. Comput. Chem. 4, 294 (1983).

${ }^{16}$ T. H. Dunning, J. Chem. Phys. 90, 1007 (1989); D. E. Woon and T. H. Dunning, ibid. 98, 1358 (1993); R. A. Kendall, T. H. Dunning, and R. J. Harrison, ibid. 96, 6796 (1992).

${ }^{17}$ M. J. Frisch, G. W. Trucks, H. B. Schlegel et al., GAussian 99, development version, Revision B.01, Gaussian, Inc., Pittsburgh, PA, 1998.

${ }^{18}$ M. E. Squillacote, R. S. Sheridan, O. L. Chapman, and F. A. L. Anet, J. Am. Chem. Soc. 101, 3657 (1979).

${ }^{19}$ S.-Y. Lee and K. F. Freed, J. Chem. Phys. 104, 3260 (1996).
${ }^{20}$ P. Celani, F. Bernardi, M. Olivucci, and M. A. Robb, J. Chem. Phys. 102, 5733 (1995).

${ }^{21}$ See EPAPS Document No. E-JCPSA6-120-311416 for vertical excitation energies (Tables S1, S4, and S7), optimized geometries (Tables S2, S5, and S8 and Figs. S1, S4, and S5) and nonvertical excitation energies (Tables S3, S6, and S9) in cis-butadiene, cZc-hexatriene, and $t E t$-hexatriene respectively, $1 \mathrm{~B}_{2} / 2 \mathrm{~A}_{1}$ conical intersection in cis-butadiene (Fig. S2) and CASSCF active orbitals in trans-butadiene (Table S3). A direct link to this document may be found in the online article's HTML reference section. The document may also be reached via the EPAPS homepage (http://www.aip.org/pubservs/epaps.html) or from ftp.aip.org in the directory /epaps/. See the EPAPS homepage for more information.

${ }^{22}$ W. Haugen and M. Traetteberg, Acta Chem. Scand. 20, 1726 (1966).

${ }^{23}$ K. Kuchitsu, T. Fukuyama, and Y. Morino, J. Mol. Struct. 1, 463 (1968).

${ }^{24}$ R. McDiarmid, Adv. Chem. Phys. 110, 177 (1999).

${ }^{25}$ O. A. Mosher, W. M. Flicker, and A. Kuppermann, Chem. Phys. Lett. 19, 332 (1973).

${ }^{26}$ O. A. Mosher, W. M. Flicker, and A. Kuppermann, J. Chem. Phys. 59, 6502 (1973)

${ }^{27}$ W. M. Flicker, O. A. Mosher, and A. Kuppermann, Chem. Phys. 30, 307 (1978).

${ }^{28}$ R. McDiarmid, Chem. Phys. Lett. 34, 130 (1975).

${ }^{29}$ R. McDiarmid, J. Chem. Phys. 64, 514 (1976).

${ }^{30}$ K. Kveseth, R. Seip, and D. Kohl, Acta Chem. Scand., Ser. A 34, 31 (1980).

${ }^{31}$ B. S. Hudson, B. E. Kohler, and K. Schulten, in Excited States, edited by E. C. Lim (Academic, New York, 1982), Vol. 6, p. 1.

${ }^{32}$ R. McDiarmid, Chem. Phys. Lett. 188, 423 (1992).

${ }^{33}$ J. P. Doering, J. Chem. Phys. 70, 3902 (1979).

${ }^{34}$ R. R. Chadwick, D. P. Gerrity, and B. S. Hudson, Chem. Phys. Lett. 115, 24 (1985).

${ }^{35}$ R. R. Chadwick, M. Z. Zgierski, and B. S. Hudson, J. Chem. Phys. 95, 7204 (1991).

${ }^{36}$ W. Fuß, W. E. Schmid, and S. A. Trushin, Chem. Phys. Lett. 342, 91 (2001).

${ }^{37}$ G. Orlandi, F. Zerbetto, and M. Z. Zgierski, Chem. Rev. 91, 867 (1991).

${ }^{38}$ L. Serrano-Andrés, J. Sanchez-Marin, and I. Nebot-Gil, J. Chem. Phys. 97, 7499 (1992).

${ }^{39}$ J. Lappe and R. J. Cave, J. Phys. Chem. A 104, 2294 (2000).

${ }^{40}$ F. Zerbetto and M. Z. Zgierski, J. Chem. Phys. 93, 1235 (1990).

${ }^{41}$ M. Aoyagi, Y. Osamura, and S. Iwata, J. Chem. Phys. 83, 1140 (1985).

${ }^{42}$ M. A. C. Nascimento and W. A. Goddard III, Chem. Phys. 36, 147 (1979).

${ }^{43}$ M. A. C. Nascimento and W. A. Goddard, Chem. Phys. 53, 251 (1980).

${ }^{44}$ R. P. Krawczyk, K. Malsch, G. Hohlneicher, R. C. Gillen, and W. Domcke, Chem. Phys. Lett. 320, 535 (2000).

${ }^{45}$ M. Ito and I. Ohmine, J. Chem. Phys. 106, 3159 (1997).

${ }^{46}$ R. L. Graham and K. F. Freed, J. Chem. Phys. 96, 1304 (1992).

${ }^{47}$ R. J. Cave, J. Chem. Phys. 92, 2450 (1990).

${ }^{48}$ R. J. Cave and E. R. Davidson, Chem. Phys. Lett. 148, 190 (1988).

${ }^{49}$ J. Y. Lee, O. Hahn, S. J. Lee, H. S. Choi, H. Shim, B. J. Mhin, and K. S. Kim, J. Phys. Chem. 99, 1913 (1995).

${ }^{50}$ P. Celani, M. Garavelli, S. Ottani, F. Bernardi, M. A. Robb, and M. Olivucci, J. Am. Chem. Soc. 117, 11584 (1995).

${ }^{51}$ B. Ostojic and W. Domcke, Chem. Phys. 269, 1 (2001).

${ }^{52}$ X. Li and J. Paldus, Int. J. Quantum Chem. 74, 177 (1999).

${ }^{53}$ J. Cabrero, R. Caballol, and J.-P. Malrieu, Mol. Phys. 100, 919 (2002).

${ }^{54}\left\langle z^{2}\right\rangle$ for trans-butadiene and $\left\langle x^{2}\right\rangle$ for cis-butadiene represent the same physical observable. The notation is different as the $z$ axis associated with the $C_{2}$ axis of the system is perpendicular to the molecular plane for the trans isomer whereas it is in plane for the cis isomer.

${ }^{55}$ S. N. Rai and R. J. Buenker, Indian J. Chem. 31A, 215 (1992).

${ }^{56}$ R. J. Cave, in Modern Electronic Structure Theory And Applications In Organic Chemistry, edited by E. R. Davidson (World, Singapore, Singapore, 1997), p. 239.

${ }^{57}$ P. G. Szalay and R. J. Bartlett, J. Chem. Phys. 101, 4936 (1994).

${ }^{58}$ W. C. Price and A. D. Walsh, Proc. R. Soc. London, Ser. A 185, 182 (1945); D. F. Evans, J. Chem. Soc. 1735, 2566 (1961); J. C. H. Hwa, P. De Benneville, and H. J. Sims, J. Am. Chem. Soc. 82, 2537 (1960); H. E. Schuler, E. Lutz, and G. Arnold, Spectrochim. Acta 17, 1043 (1961).

${ }^{59}$ R. M. Gavin, S. Risemberg, and S. A. Rice, J. Chem. Phys. 58, 3160 (1973); R. M. Gavin and S. A. Rice, ibid. 60, 3231 (1974).

${ }^{60}$ F. W. Knoop and L. J. Oosterhoff, Chem. Phys. Lett. 22, 247 (1973).

${ }^{61}$ N. G. Minnaard and E. Havinga, Recl. Trav. Chim. Pays-Bas 92, 1179 (1973). 
${ }^{62}$ D. E. Post, W. M. Hetherington, and B. Hudson, Chem. Phys. Lett. 35, 259 (1975).

${ }^{63}$ W. M. Flicker, O. A. Mosher, and A. Kuppermann, Chem. Phys. Lett. 45, 492 (1977)

${ }^{64}$ D. H. Parker, S. J. Sheng, and M. A. El-Sayed, J. Chem. Phys. 65, 5534 (1976); D. H. Parker, J. O. Berg, and M. A. El-Sayed, Chem. Phys. Lett. 56, 197 (1978).

${ }^{65}$ A. J. Twarkowski and D. S. Kliger, Chem. Phys. Lett. 50, 36 (1977).

${ }^{66}$ J. P. Doering, A. Sabljic, and R. McDiarmid, J. Phys. Chem. 88, 835 (1984).

${ }^{67}$ A. Sabljic and R. McDiarmid, J. Chem. Phys. 82, 2559 (1985).

${ }^{68}$ A. Sabljic and R. McDiarmid, J. Chem. Phys. 84, 2062 (1986).

${ }^{69}$ W. J. Buma, B. E. Kohler, and K. Song, J. Chem. Phys. 92, 4622 (1990); 94, 6367 (1991)

${ }^{70}$ H. Petek, A. J. Bell, R. L. Christensen, and K. Yoshihara, J. Chem. Phys. 96, 2412 (1992)

${ }^{71}$ T. Fujii, A. Kamata, H. Shimizu, Y. Adachi, and S. Maeda, Chem. Phys. Lett. 115, 369 (1985).

${ }^{72}$ S. Lochbrunner, W. Fuß, K.-L. Kompa, and W. E. Schmid, Chem. Phys. Lett. 274, 491 (1997).

${ }^{73}$ C. Woywod, W. C. Livingood, and J. H. Frederick, J. Chem. Phys. 112, $613(2000)$.
${ }^{74}$ C. Woywod, W. C. Livingood, and J. H. Frederick, J. Chem. Phys. 114, 1631 (2001).

${ }^{75}$ D. J. Leopold, R. D. Pendley, J. L. Roebber, R. J. Hemley, and V. Vaida, J. Chem. Phys. 81, 4218 (1984).

${ }^{76}$ C. W. Bock, Y. N. Panchenko, S. V. Krasnoshchiokov, and V. I. Pupyshev, J. Mol. Struct. 148, 131 (1986), and references therein.

${ }^{77}$ F. W. Langkilde, R. Wilbrand, O. F. Nielsen, D. H. Christensen, and F. M. Nicolaisen, Spectrochim. Acta, Part A 43, 1209 (1987).

${ }^{78}$ M. Traetteberg, Acta Chem. Scand. 22, 2294 (1968).

${ }^{79}$ X. Ci and A. B. Myers, J. Chem. Phys. 96, 6433 (1992).

${ }^{80}$ S. Sakai and S. Takane, J. Phys. Chem. A 103, 2878 (1999).

${ }^{81}$ M. Garavelli, P. Celani, F. Bernardi, M. A. Robb, and M. Olivucci, J. Am. Chem. Soc. 119, 11487 (1997).

${ }^{82}$ K. Ohta, Y. Naitoh, K. Tominaga, N. Hirota, and K. Yoshihara, J. Phys. Chem. A 102, 35 (1998).

${ }^{83}$ Analytical energy gradients have recently been implemented for secondorder multireference perturbation theory (MRPT2): P. Celani and H.-J. Werner, J. Chem. Phys. 119, 5044 (2003). However, in cases with strong valence-Rydberg mixing like butadiene and hexatriene, a multistate treatment is required, which allows a relaxation and mixing of the zeroth-order wave functions in the correlation treatment. MRPT2 analytical energy gradients are not yet implemented in such cases. 